\title{
MASS LOSS FROM STARS AND THE LOCATION OF BLUE SUPERGIANTS IN THE HR DIAGRAM*
}

\author{
(Abstract)
}

\author{
Yu. FRANTSMAN and E. POPOVA
}

Radio Astrophysical Observatory, Latvian Academy of Sciences, Riga, U.S.S.R.

Continuing the discussion about the evolution of massive stars, about cases A and B of the computations of evolution, I want to say some words about the comparison of the observations and theoretical computations.

As it is known, on the HR diagram blue supergiants occupy the effective temperature region between $3.9<\log T_{e}<4.35$.

In case $B$, the Schwarzschild stability criterion, the star exhausts the main part of its He-fuel as a blue supergiant. The core He-burning models are placed in $\log T_{e}$ region between 3.8 and 4.2. This is in good agreement with observations. The computations for the case $\mathrm{A}$, Ledoux criterion, give the effective temperature only in the narrow region $\left(4.15<\log T_{e}<4.25\right)$. This does not agree with the observational data. But these calculations of stellar evolution were performed assuming that mass loss does not occur.

The question is: if the mass loss from the star in the previous evolutionary stage, red supergiants, may give greater dispersion of the effective temperature for blue supergiants. Sequences of inhomogeneous equilibrium models of stars with a constant mass of the He-core and decreasing mass of the $\mathrm{H}$-rich envelope are calculated for stars with central helium burning in the blue supergiant region. The initial masses of the sequences are $15 \mathfrak{M}_{\odot}$ and $30 \mathfrak{M}_{\odot}$. Such models may represent the stars, which lost some amount of mass in the red supergiant region.

The chemical composition distribution was taken according to case $\mathrm{A}, X=0.70$ in the envelope, $X=0$ in the core. It was shown that continuous sequences of models with decreasing mass of the hydrogen-rich envelope can be obtained in the blue supergiant region.

The effective temperature of these models depends on the amount of mass lost in the red supergiant stage, or equivalently the mass of the hydrogen envelope.

\section{Conclusion}

If a massive star loses up $20-30 \%$ of its mass in the red supergiant stage it returns to the blue supergiant region in HR diagram where the blue supergiants really are observed.

Then, in this point of view, there is no discrepancy between observational data and computations in case $\mathrm{A}$.

* This paper was presented by Yu. Frantsman. 